The American Journal of Bioethics (forthcoming)

\title{
Habits, Nudges, and Consent
}

\section{Ezio Di Nucci (Universität Duisburg-Essen)}

In any health care crisis, the first priority is to improve the patient's condition. But this priority is not absolute as it is normally subject to the patient's (or someone on her behalf) approval. This can sometimes lead to serious difficulties when the patient - on epistemological (the patient may be ignorant or misinformed), political (the patient may have political objections to a particular treatment or medical professional), emotional (the patient may be just too scared), ethical (see 'political'), personal (the patient may want to die or suffer), or religious (see 'political') grounds - refuses treatment. The compatibility of successful medical treatment and patient's consent - and the extent to which medical paternalism can be justified - is one of the great challenges of contemporary medical ethics.

It has been recently suggested (Cohen, forthcoming) that we can make progress on this issue by showing that the choice architecture of nudges (Thaler \& Sunstein, 2008) is compatible with informed consent, so that we can obtain 
better patient choices without violating patients' autonomy by nudging them in the right direction.

This proposal raises two distinct issues: $(I)$ one is the issue of whether nudges really can be compatible with informed consent. The other issue - which is just as important - is (2) whether nudges can be compatible with informed consent while at the same time effectively improving patient choices. I say that this latter question is just as important because it would be no great progress (not in the real world, anyway) if the only compatible nudges were weak and ineffective ones.

I am sympathetic to Cohen's discussion and to nudges more in general, but I see a problem with the point just mentioned about the effectiveness of nudges: Cohen's discussion is incomplete in one important respect (as he himself admits, see for example page 22): his argument is only that nudges must not be, in principle, incompatible with informed consent because there are at least some legitimate nudges that are not incompatible with informed consent. Now, the question of what counts as a legitimate nudge is not a very interesting one: for one, the concept is too young for a scholarly history of it; and, more importantly, as long as $\mathrm{X}$ can effectively improve wellbeing non-coercively, then the question of whether $X$ should count as a real nudge in Thaler\&Sunstein's sense is merely 
academic (in its pejorative sense). But there is a non-academic point here: it must not be the case that all the really effective nudges are autonomy-violating and that the only nudges that are compatible with consent aren't that effective exactly in virtue of their compatibility with consent: otherwise the theoretical point that Cohen argues against - namely that there is conceptual tension between nudges and consent - would end up being vindicated.

Nudges owe much of their effectiveness to the less than fully conscious character of their influence: think of the original example of a nudge which opens Thaler\&Sunstein's book (2008: I): the ordering and placing of food in a cafeteria. The point is that, without our realizing, variables such as (i) exactly where chips are (relative to green beans, say), (ii) how high chips are on the shelves, (iii) how well illuminated chips are or (iv) how they are packaged, make a difference to the probability of our choosing chips (both in general and, say, over green beans). This difference in probability need not be a significant one to make a contribution to health and wellbeing when considered across the whole life of one individual or across the lives of an entire population. If the difference were more significant, it would probably be or soon become conscious. And if the difference became conscious, then it would be at risk because it would be more easily exposed to the motives that lead us to chips in the first place. So the influence of 
nudges needs to remain less than conscious to remain relevant to wellbeing, which is another way of saying that nudges work best when less than conscious. In this respect, the real challenge is to show that less than conscious nudges are also compatible with informed consent, because those are the nudges which could make considerable contributions to improving the health and wellbeing of entire populations.

A further distinction needs to be made which helps to explain the effectiveness of nudges: what is crucially less than conscious isn't just the increased probability effect, it is also what increases the probability: so that in Thaler\&Sunstein's original cafeteria example, subjects are not just unaware of the decreased probability of their eating chips resulting from chips' new place in the cafeteria; subjects are also unaware of the very fact that chips are now lower down on the shelves and therefore ever so slightly harder to grab. This is crucial because it distinguishes Thaler\&Sunstein's original nudge from the kinds of nudges that Cohen uses to make the case that nudges are not incompatible with informed consent, such as for example increasing the amount of caloric information on food packaging. Supposedly, increasing caloric information on food packaging also makes a difference to the probability of the relevant food consumption. Also, the further similarity with Thaler\&Sunstein's original nudge is 
that consumers may very likely be similarly unaware of this statistical fact as they are unaware of the relevant statistical facts in the cafeteria case. But the crucial difference is that consumers won't be unaware of the new caloric information if that's to make a difference to their buying behaviour: they need to read it!

The general point is that not all choices and actions that can be influenced in the subject's own interest are of the same kind: some of them are habitual, automatic, and unaware - such as reaching up to grab a can on a shelf. Others are deliberative and conscious, such as reading what it says on that can. This crucial theoretical difference is interesting in itself and poses all sorts of questions in the philosophy of action, philosophy of mind, and philosophy of psychology: are habitual and automatic behaviors proper actions? Are they intentional? Can these be explained by appeal to psychological reasons? Are we free when acting habitually and automatically? Are we responsible for those less than conscious kinds of actions? (I have discussed these and related questions at length elsewhere - see Di Nucci 2008, Di Nucci 20Ila, Di Nucci 20I Ib, Di Nucci 2012, and Di Nucci forthcoming). There has been a lot of interest from empirical psychologists on those and related phenomena, especially in the priming literature that has seen an exponential growth since John Bargh's first experiments in the '90s (Bargh et al. 1996). And recently this work has also 
come to the attention of the wider public with more popular publications such as Gladwell's Blink, Kahneman's Thinking, Fast and Slow and indeed also Thaler \& Sunstein's Nudge. The relevance of research on less than conscious judgment and behaviour, here, is that these phenomena enable nudging: and it is (also) these phenomena - rather than (only) conscious propositional cases such as Cohen's example of adding caloric information on food packaging - that need to be shown to be compatible with informed consent if nudging in health care is to represent a significant improvement in the real world.

To put the point somewhat crudely: can we still talk about informed consent if nudging means influencing agents in a certain direction without their realizing it? There is an obvious conceptual tension between informed consent and lack of awareness because the whole point of obtaining informed consent is to inform the patient, namely to make her aware of what is going on. But I think that, at least sometimes, this tension can be resolved. Cohen's example of a surgeon's optimism is a case in point here: the patient doesn't need to consciously notice the surgeon's optimism for the surgeon's attitude to have a positive effect on the probability of the agent's consenting to surgery. Also, there are forms of consent that do not necessarily presuppose awareness: there seems to be a difference between having a consenting attitude that does not get to be expressed (tacit 
consent) and lacking a dissenting attitude; the latter can, presupposing perceptual experience of the relevant change in the environment, also be enough for consent (implicit consent). If we can talk about consent even in cases such as this last one where there is neither a consenting attitude nor a consenting action, then we no longer need to necessarily appeal to the person's psychology in order to talk about consent - and then the conceptual link between subjective awareness and consent would have at least been weakened.

In conclusion, then, we need to distinguish between easy and hard cases when talking about the compatibility of nudging with informed consent: propositional conscious cases such as Cohen's adding caloric information to packaging can be easily showed to be compatible with informed consent, but these cases miss out on the real power of nudging: its appeal to less than conscious mechanisms. And in order to make those less than conscious nudges generally compatible with consent we may have to appeal to a more diversified conception of consent. 


\section{References}

I. Bargh JA, Chen M, \& Burrows L. 1996. Automaticity of Social Behavior: Direct effects of trait construct and stereotype activation on action. Journal of Personality and Social Psychology 7I:230-244.

2. Cohen S. Nudging and Informed Consent. American Journal of Bioethics (forthcoming).

3. Di Nucci E. 2008. Mind Out of Action. VDM.

4. Di Nucci E. 20I la. Automatic Actions: Challenging Causalism. Rationality Markets and Morals 2(I):179-200.

5. Di Nucci E. 20l Ib. Frankfurt versus Frankfurt: a new anti-causalist dawn. Philosophical Explorations I4(I):I17-131.

6. Di Nucci E. 2012. Priming Effects and Free Will. International Journal of Philosophical Studies 20(5):725-734.

7. Di Nucci E. Mindlessness. CSP (forthcoming).

8. Gladwell M. 2005. Blink. Little, Brown.

9. Kahneman D. 20I I. Thinking, Fast and Slow. Farrar, Straus and Giroux.

I0. Thaler H.T. \& Sunstein C.R. 2008. Nudge. Yale UP. 